\section{Effect of emotion regulation training on depression, anxiety, and stress among mothers of children with mental disorders}

Hossein Jenaabadi ${ }^{1}$

\begin{abstract}
Not only mental disorders do affect patients' efficiency, but also they have adverse impacts on families of suffered patients through disrupting their performance. This study aimed to compare the effect of emotion regulation training based on Gross process model and Linehan's dialectical behavior therapy in depression, anxiety, and stress among mothers of children with mental disorders. This was a quasi-experimental study designed as pretest-posttest with a control group. 27 mothers of children with depression and anxiety disorders who admitted to the rehabilitation centers and clinics was selected. They were allocated to three groups including the emotion regulation training based on the Gross's model, the Linehan's dialectical behavior therapy, and the control group. The experimental groups were trained during 8 sessions of 120-150 min. However, the control group received no such interventions. Using the lovibond and lovibond depression, anxiety, and stress scale. The results indicated that there were no significant differences between the mothers placed in the experimental groups; however, compared to the control group, significant decreases in the measured variables were observed in the experimental groups). The emotion regulation training program can be considered as a part of intervention programs conducted at counseling centers and public hospitals for mothers of children with special needs in order to promote mental health and decrease the emotional pain and suffering in the involved family members.
\end{abstract}

Keywords: Behavior Therapy, Emotion, Mental Disorders

\section{Introduction}

Not only mental disorders do affect patients' efficiency, but also they greatly influence families of suffered patients through disrupting their performance. This impact can be observed in various aspects including mental health and social, economic performance. Overall, mental disorders decrease a family's level of well-being and they impose great pressure on the family members [1]. When a family member suffers from a mental disorder, all family members somehow have to deal with the disease and its consequences [2]. Being in a family who has a member with mental disorders often imposes major pressure on other family members [3].

Several research evidences have shown that parents of children with special needs experience increasing levels of parental stress 
and anxiety compared to parent of healthy children [4-6]. However, this effect varies according to the nature of the child's disorder and some family variables. Sudden disorders that have an acute start, in comparison with chronic disorders, have different effects on family life. Moreover, a disorder may issue different challenges to the family in various stages and as the child grows, his/her behaviors become more challenging [7].

When dealing with various conditions, each person has a certain level of stress threshold. When too much pressure is on a person, mental and emotional disorders inevitably occur [8]. A type of stress, that can cause serious mental pressure, put the foundations of family at risk, and have long-term effects on the family, is the stress caused by having a sick child who suffers from long-term chronic disorders [9]. Although having a child makes the mother feel pleasure, pride, and personal growth, it has its own challenges and this is why it is stressful [10]. These challenges can be accompanied by negative complications, such that these parents compared to those who do not have any children, experience more levels of anxiety and depression [11].

In our society, mothers, compared to fathers, due to various reasons such as spending more time with their children, are more involved in matters related to their children. As a result, they interact more with their children and endure a lot of stress [12]. Mothers (women) are exposed to two major kinds of stress; the stress caused by biological identity and the stress caused due to professional responsibilities. Women's reproductive action is the basic stress syndrome in women; another source of stress in women is related to their professional and housekeeping responsibilities. Taking care of the elderly, children, especially those with special needs, are among the later kind of stress [13]. Therefore, mothers of children with mental disorders experience higher levels of stress compared to mothers of normal children [14]. Through comparing the level of stress among children's parents, Gupta concluded that parents of children whose disorders are accompanied with more behavioral symptoms experienced higher levels of stress [15]. Based on what was mentioned, it can be concluded that through examining and comparing the level of stress among parents of children with mental disorders, the priority of therapeutic interventions in these parents can be determined and many of these parents' issues related to parenting can be prevented using parenting stress management interventions. There is a positive relationship between the occurrence of negative stress and the onset of depression and nearly 20 to 50 percent of people who experience severe stress suffer from depression [16]. Depression is the most common mental disorder among mothers of children with mental disorders [17], especially developmental disorders [12]. Behavioral problems of children with mental disorders, particularly their disability in doing their personal chores, cause stress in their mothers [1].

Depression often starts from a situation where changes happen in a person's life and the person has to abandon all the important people and issues related to his/her life such as workplace and home [1].

Since about 15 percent of people with major depression commit suicide [18] and given the prevalence, long-term effects of child's disability on these children's family life, the possibility of having suicidal thoughts and attempting to commit suicide increases [19]. According to the conducted research, emotional problems such as depression, anxiety, hostility, stress, and interpersonal sensitivity are more prevalent among mothers of children with mental disorders compared to other populations [8]. Studies carried out in Iran revealed that mood disorders, compared to other disorders, are more prevalent among women (mothers) [20].

In fact, emotion regulation refers to people's attempt to influence the type, time, and the method of gaining experiences and expressing them, to change the duration or severity of cognitive, behavioral, and/or physical emotion processes [21]. Emotion 
regulation has attracted researchers' attention as one of the psychological variables [22] which plays a significant role in people's adaptability with stressful life events [23]. Furthermore, emotion regulation has a leading role in the development and maintenance of emotional disorders; it includes a wide range of physiological and behavioral conscious and unconscious cognitive processes [24], and refers to the cognitive styles used by each person to increase or decrease and/or retain his/her emotions [25].

The study conducted by Sartorious, et al. [7] indicated that emotion regulation training affected the mental health of people who deal with chronic diseases. Clarkin et al, [26], Soler et al. [27], and McQuillan et al. [28] examined the effect of training the skills of Linehan's dialectical behavior therapy and the skills of Gross's therapy and indicated the effect of these therapies on depression, anxiety, and other variables of mental health. In addition, Miller et al. [29], Vanden, Bosch, [30], and Karbalaei [31] demonstrated the effects of this therapy on decreasing impulsivity, increasing emotion regulation, and improving mood and emotional issues such as depression, anxiety, anger, emotional instability, and excitability.

Emotion regulation is examined within two main frameworks including emotion regulation strategies which are activated before or at the beginning of the incident of an event and emotion regulation strategies that are activated after an accident happened or after the formation of the emotion. Emotion regulation strategies activated before the incident of an event play an important role in controlling negative emotions [32], since they somehow interpret the event in a way that they decrease the negative emotional responses. Studies indicated that differences in the use of various cognitive emotion regulation styles led to various emotional, cognitive, and social consequences, such that using reappraisal styles are associated with positive emotions, better interpersonal functions, and higher well-being [24]. Given the role of emotion regulation difficulties in creating emotional issues, it seems that training and applying emotion regulation skills and comparing the separate effects of these two methods in reducing emotional problems can help expand the scope of treatment; therefore, the present study was conducted to examine the effect of emotion regulation training based on the Gross process model and linehan's dialectical behavior therapy on mental health (depression, anxiety, and stress) among mothers of children with mental disorders.

\section{Method}

In the quasi experimental study, pretestposttest design with a control group was employed. The statistical population included all mothers of children with mental disorders (developmental disorders, emotional or internalizing disorders, and disruptive behavior or externalizing disorders) admitted to the rehabilitation centers and clinics in Zahedan, the east of Iran. Through holding a group briefing (including explaining the main objectives), the people who were willing to participate in this study were identified and after obtaining their consent, they were asked to complete the questionnaire. People who suffered from depression and anxiety disorders, obtained a score 1 standard deviation higher than the mean score, and those who referred to psychiatrists once or twice a month were placed in the experimental groups. The partisipants included 27 mothers of children with mental disorders (developmental disorders, emotional disorder, and disruptive behavior disorder). After holding a briefing meeting and obtaining the participants' oral commitment to stop the drug treatment and to take part in the training sessions, the number of participants reduced to 21 partisipants, who were placed in three groups including emotion regulation training based on the Gross's process model, emotion regulation training based on the Linehan's dialectical behavior therapy, and a control group.

The inclusion criteria included having the diagnostic criteria of mental disorders (developmental disorders, emotional 
disorders, and disruptive behavior disorders) in children, having at least diploma degree, being in the age range of 25 to 33 years old, not receiving any concurrent psychological therapies aiming at treating anxiety, depression, and stress disorders, and having a child aged 2 to 8 years old with mental disorders. After obtaining their consent, people who had the abovementioned criteria completed a questionnaire on depression, anxiety, and stress. Exclusion criteria included mothers with more than one child with special needs, divorced or widowed women, women working outside the house, patients undergoing psychotherapy, and women who were the head of household.

In the current study, two methods of training were used: emotion regulation training based on the Gross's model and the Linehan's dialectical behavior therapy. The most important differences between these two methods are as follows. First, according to the dialectical behavior therapy, a person who deals with an emotional problem does not have the ability to perceive the totality of emotions and experiences and he/she mingles with them and has a problem in distancing himself/herself from the problem. Therefore, developing the mindfulness and living in the moment, which constitute an important part of the skills, aid people to distance from emotions, understand the totality of the problem, and finally dominate the problem. Second, in other behavioral and cognitive approaches (including emotion regulation training based on the Gross's model), the main focus is on the change; however, in the dialectical behavior therapy, the main emphasis is on the amount of change and even, more than that, on the acceptance and validating emotions [33]. Before starting the experiment, instructions for holding 8 training sessions of 2-2 $1 / 2$ hours were adjusted. In each session, 90 minutes of time spent on training skills considered for that session and the remaining time on reviewing the taught skills and skills which should be practiced at home. The sessions were held as group therapy. During the training sessions, basic skills and advanced emotion regulation skills were taught. These skills include training how to identify and become familiar with emotions, how to fill out the worksheet on identifying emotions, thoughts, and automatic feelings, how to practice recording emotions through saying them out loud (shouting the feelings), how to identify barriers to healthy emotions (emotions, behavior, and thoughts), how to become familiar with emotions which lead to risky behaviors, training cognitive behavior therapy skills (encouragement, rewarding the behaviors, repeating the action), training and attempting to reduce the physical vulnerability to the turbulent emotions (eating, bulimia and anorexia, exercise, illness and physical pain, physical tension, and stress), training how to practice identifying self-injurious behaviors, how to find oneself without considering others' judgment based on a form completed at home, how to decrease cognitive vulnerability, how to practice the paradox of thought and emotion, and how to use coping thoughts, providing a list of coping thoughts, training the balance between thoughts and feelings using the form, training and practicing a form of recording the evidence to consider all aspects, training how to practice planning an enjoyable experience and how to apply a form to record enjoyable activities, practicing mindfulness of emotions without judging the emotions (focusing on breathing, focusing on the current and past emotions, paying attention to somatic sensations related to emotions, naming emotions, subjective imagination, observing experienced emotions, talking to oneself about the feelings, observing experienced judgments, and conscious breathing), facing the emotions, practicing how to record emotions and fill out the emotion form, training and practicing how to deal with emotions, training how to act against extreme emotional desires and how to complete the worksheet on planning opposite action, training and practicing how to complete the form on behavior analysis, training problem solving skills, training how to find a solution and how to use weekly form used to record 
the emotions to reduce the vulnerability and observing and accepting emotions [23,34,35]. The Lovibond and Lovibond Depression, Anxiety, and Stress Scale [36] were used for data collection. This self-report scale is composed of 3 subscales which evaluate three negative emotional states including depression, anxiety, and stress. The main application of this scale is to measure the severity of symptoms of depression, anxiety, and stress. To fill out this scale, a person should determine the status of a symptom during the week prior to the time the study is conducted. Since this scale could provide a comparison of the differences among the severity of symptoms during various weeks, it can be applied to assess the treatment progress over time. Antony, Beiling, Cox, Enns, and Swinton [37], using factor analysis, concluded that this scale has three factors including depression, anxiety, and stress. Alpha coefficients of depression, anxiety, and stress were respectively $0.92,0.97$, and 0.95 .

Moreover, the results of calculating the correlation among these factors conducted by Antony et al. showed that the correlation coefficient between depression and stress was 0.48 and the correlation coefficient between anxiety and stress was 0.53 , and the correlation coefficient between anxiety and depression was 0.28 . The reliability and validity of this scale was examined by Samani and Jokar [38]. They reported that the test-retest reliability of depression, anxiety, and stress was respectively $0.80,0.76$, and 0.77 and the Cronbach's alpha coefficients of depression, anxiety, and stress were respectively 0.81 , 0.74, and 0.78. Each subscale includes 7 questions and the final score for each subscale is achieved through summing all scores related to that subscale. Each question is scored from 0 (does not apply to me at all) to 3 (applies to me most of the time). Since DASS-21 is the short-form of the original scale (42 items), the final score for each subscale should be multiplied by two and the symptoms and severity of each subscale are explained in 5 levels (normal, mild, moderate, severe, and extremely severe) [39].

\section{Results}

Since the participants obtained two scores (in the pretest and posttest), to analyze the obtained data, Multivariate analysis of covariance (MANCOVA) was used with an independent variable, i.e. the group of admission (experimental and control groups), an auxiliary variable, i.e. the pretest scores, and a dependent variable, i.e. the posttest scores of depression, anxiety, and stress subscales. Overall, 21 individuals participated in this study. Each group of experimental (emotion regulation training based on the Gross's model and the Linehan's dialectical behavior therapy) and control included 7 partisipants. The age range of participants was 25 to 33 years old. The mean age of the emotion regulation training based on the Gross's model, the Linehan's dialectical behavior therapy, and the control group was respectively 27.54, 26.94, and 27.06. The minimum level of education was high school diploma. The severity and type of disorders from which the participants' children suffer were not under study and these variables were not controlled. To investigate the homogeneity among the experimental and control groups, using the multivariate analysis of variance (MANOVA), the participants were randomly allocated to the groups based on mental health in the pretest.

Considering the primary level and regarding the results in the pretest as the basis, the results indicate that there are no significant differences between the control and experimental groups in terms of depression $(\mathrm{F}(18,2)=2.51, \mathrm{p}=0.07)$, anxiety $(\mathrm{F}(118,2)=$ $2.14, \mathrm{p}=0.09)$, and stress $(\mathrm{F}(18,2)=0.83$, $\mathrm{p}=0.21)$. This indicates the homogeneity of the experimental and control groups with regard to the dependent variable in the pretest. Factor analysis of covariance was conducted to examine the differences in subscales of stress, anxiety, and depression between mothers placed in the experimental groups and those in the control group. The results of Box's $M$ confirm the assumption of homogeneity of the variance-covariance 
matrix $(\mathrm{F} \quad(1092,14.08)=0.54, \quad \mathrm{p}>0.05)$. The results of Levene's test, conducted to test the assumption of homogeneity of the groups' variance, indicate that considering all three subscales, i.e. stress, anxiety, and depression, the experimental and control groups were homogeneous in terms of variance.

Table 1 Descriptive statistics related to depression, anxiety, and stress obtained in the pretest and posttest

\begin{tabular}{|c|c|c|c|c|c|c|c|}
\hline Groups & Subscales & $\begin{array}{c}\text { Mean in the } \\
\text { pretest }\end{array}$ & $\begin{array}{l}\text { Standard } \\
\text { deviation }\end{array}$ & $\begin{array}{c}\text { Mean in the } \\
\text { posttest }\end{array}$ & $\begin{array}{l}\text { Standard } \\
\text { deviation }\end{array}$ & $\begin{array}{c}\text { Adjusted } \\
\text { mean }\end{array}$ & $\begin{array}{c}\text { Standard } \\
\text { error }\end{array}$ \\
\hline \multirow{2}{*}{$\begin{array}{l}\text { Gross's } \\
\text { model }\end{array}$} & Depression & 26.76 & 0.91 & 15.23 & 1.36 & 14.31 & 0.31 \\
\hline & Stress & 24.42 & 0.82 & 17.81 & 1.21 & 15.18 & 0.38 \\
\hline \multirow{2}{*}{$\begin{array}{l}\text { Linehan's } \\
\text { model }\end{array}$} & Depression & 25.73 & 0.72 & 15.71 & 1.12 & 16.13 & 0.52 \\
\hline & Stress & 24.23 & 1.27 & 19.18 & 0.92 & 20.02 & 1.12 \\
\hline \multirow{3}{*}{$\begin{array}{l}\text { Control } \\
\text { group }\end{array}$} & Depression & 25.53 & 2.21 & 23.21 & 1.92 & 14.94 & 0.47 \\
\hline & Anxiety & 24.31 & 1.32 & 21.01 & 1.13 & 14.17 & 0.62 \\
\hline & Stress & 25.17 & 0.82 & 25.82 & 0.91 & 26.00 & 1.21 \\
\hline
\end{tabular}

The results of analysis of covariance (ANCOVA), conducted to examine the effect of group therapy on the considered subscales through controlling the effect of the pretest as a factor affecting the posttest, indicate a significant decrease in the levels of depression, anxiety, and stress among mothers placed in the experimental groups compared to those in the control group (Table 2). Examining the results of Bonferroni's post hoc test reveal that there are no significant differences between the emotion regulation training based on the Gross's process model and the Linehan's dialectical behavior therapy in terms of depression, anxiety, and stress; however, both groups indicate a significant improvement compared to the control group $(\mathrm{p}<0.001)$.

\begin{tabular}{|c|c|c|c|c|}
\hline Subscale & Degree of freedom & $\mathrm{F}$ & $\begin{array}{c}\text { Level of } \\
\text { significance }\end{array}$ & Eta squared \\
\hline Depression & $2-15$ & 117.03 & 0.001 & 0.98 \\
\hline Anxiety & $2-15$ & 36.78 & 0.001 & 0.91 \\
\hline Stress & $2-15$ & 47.93 & 0.001 & 0.84 \\
\hline
\end{tabular}

\section{Discussion}

The results of this study indicated that two methods of training emotion regulation skills in the same way led to a stable decrease in the symptoms of depression, anxiety, and stress. This is probably due to the fact that the main emphasis of emotion regulation methods is on mindfulness, controlling negative emotions and modification, practical exercises and workshops, holding group sessions, and thus, coping with emotional ambivalence. Considering the participants' scores in the pretest and posttest, in comparison with the Gross's process model, after conducting the emotion regulation training based on the dialectical behavior therapy, the participants' scores on depression significantly decreased. This confirmed the effect of the dialectical behavior therapy on depression.

According to the principles of the dialectical behavior therapy, a therapist should consider the impact of information processing systems on emotional, biological, and social regulations. Emotional irregularity influences the cognitive restraint, contributes to the inability to solve a problem, and makes the situation out of control. In the second level, the therapist should carefully examine the mutual effects of family, culture and other 
environmental factors. The patients may learn the effective communication skills; however, they may be mocked and blamed as soon as they use these skills. Therefore, in this therapy, the therapist should have a comprehensive awareness of the patients' problem. Linehan believes that patients' problems stem from the fact that these patients cannot solve the conflicts without setting aside their polarized attitudes towards the issues and they think that they should act either based on external factors or internal ones [2]. In fact, the dialectical behavior therapy aids patients to reach a harmony and makes inconsistent matters consistent with each other. One of the interesting points about the dialectical behavior therapy is the difference it made between emotional pain and emotional suffering. Sometimes, training on the dialectical behavior therapy skills emerges the following question: is it possible to feel nothing when dealing with the problems and difficulties of life? Emotional pain is a natural reaction to the problems of life; however, the issue is created when people are not willing or are not able to accept the natural emotional pain. In this case, people are willing to fight it and get rid of the problem very quickly. In such a condition, emotional pain changes into emotional suffering [34].

The caregivers of children with mental disorders, due to their children's permanent condition, their age and constant needs, suffer from emotional suffering. This is an unnatural long lasting reaction that makes the situation worse and involves all the people. Therefore, the main objective of the dialectical behavior therapy is to alleviate the suffering of people involved in the emotional problems. To achieve this goal, in the current study, distress tolerance and emotion regulation skills were taught. Selecting people whose emotional pain had changed into emotional suffering was among the innovations of the current study.

In the dialectical behavior therapy, the clinical specialists are asked to help the clients perceive disruptive behaviors as acquired behaviors to solve their problems. The client does not have essential skills to react to more creative methods. This is why the dialectical behavior therapy is effective in reducing the tendency towards depression. This finding is in line with the results of Linehan [40], Vanden Bosch, Verhuel, Schippers, and Brink [30], and Kroger et al. [11] who indicated the effect of the dialectical behavior therapy and group training in decreasing self-injurious behaviors and emotion regulation and improving a number of mood and emotional issues including depression, anxiety, anger, emotional instability, and excitability among the people under the subject of study [31].

Both training methods decreased symptoms of anxiety; however, the method conducted based on the behavior therapy led to a greater decrease in the participants' scores in the posttest. This can be due to the fact that this therapy is a more comprehensive therapy and it emphasizes various situations and acceptance and change along each other. Moreover, the dialectical behavior therapy focuses on the deeper layers of emotions [30]. This finding is in line with the results of Miller et al. [29], Vanden Bosch, Verhuel, Schippers, and Brink [30], and Kroger et al. [11] who indicated the effect of the dialectical behavior therapy and group training in decreasing self-injurious behaviors and emotion regulation and improving a number of mood and emotional issues including depression, anxiety, anger, emotional instability, and excitability among the people studied. Overall, the results indicated that emotion regulation training based on the dialectical behavior therapy led to a decrease in stress, so that the level of stress among participants placed in this group decreased more compared to the level of stress among participants in the other experimental group whom were under training based on the Gross's process model. This finding is consistent with the results of Zamani and Habibi [1] who demonstrated that the Linehan's emotion regulation training decreased the level of stress among mothers of children with mental disorders. A study the results of which reject these findings was not found. 


\section{Conclusion}

The results indicated that there were no significant differences between mothers placed in both experimental groups considering the levels of stress, anxiety, and depression; however, compared to the control group, significant decreases were observed in the experimental groups. Therefore, emotion regulation training can be considered as a part of therapeutic intervention conducted at counseling centers and public hospitals for the mothers of children with special needs to promote mothers' mental health.

\section{Acknowledgements}

The author would like to thank all the individuals who participated in this study.

\section{Contribution}

Study Design: HJ

Data Collection and Analysis: HJ

Manuscript Preparation: HJ

\section{Conflict of Interest}

"The authors declare that they have no competing interests."

\section{Funding}

The author (s) received no financial support for the research, authorship and/or publication of this article.

\section{References}

1- Zamani N, Habibi M. Compare the influence of both dialectic and cognitive behavior therapies to maintain mothers' mental health whose children with Special needs. Journal of Research and Health. In Press.

2- Koegel RL, Schreibman L, Loos LM, et al. Consistent stress profile in mothers of children with autism. $J$ Autism Dev Disord1992; 22(2): 205-16.

3- Barlow DH, Durand VM. Abnormal psychology. Belmont: Wadsworth; 2002.

4- Dowdell EB. Caregiver burden: grandmothers raising their high risk grandchildren. J Psychosoc Nurs Ment Health Serv1995; 33(3): 27-30.

5- Hankin CS. ADT-ID and its impact on the family. Drug Benefits Trends2001; 13: 15-6.

6- Sanders JL, Morgan SB. Family stress and adjustment as perceived by parents of children with autism or Down syndrome: Implications for intervention. Child and Family Behavior Therapy1997; 19: 15-32.

7- Kuipers E, Bebbington PE. Families and mental disorders: from burden to empowerment. In: Sartorius N, Leff J, López-Ibor JJ, Maj M, Okasha A, eds. John Wiley and Sons Ltd; 2004.

8- Hakimjavadi M, Lavasani MGH, Haghighatgi M, Zebardast O. Relationship among depression, anxiety, stress and personality in veteran children. Iranian Journal of Public Health2010; 3(1): 9-16.

9- Sajedi F, Vameghi R, Alizad V, et al. Is Anxiety More Common in Mothers of children with Cerebral Palsy? The Journal of Rehabilitation2011; 11(5): 15-20.

10- Ostberg M. Parental stress, psychosocial problems, and responsiveness in help seeking parents with small (2-45 months old) children. Acta Paediatr 1998; 87(1): 69-76.

11- Kröger C, Schweiger U, Sipos V. Effectiveness of dialectical behavior therapy for borderline personality disorder in an inpatient setting. Behav Res Ther2006; 44(8): 1211-7.

12- Zamani N, Zamani S, Habibi M. Comparison of stress in mothers caring for children with developmental disorders, External and internal disorders and children with normal. Journal of Research \& Health. In Press. 13- Khodayarifard M, Parand A. Stress and coping skills in adolescents. Tehran: Tehran University Publication; 2007.

14- Habibi Asgarabad M, Rashidi A, Motovalipour A. Parenting stress in mothers of exceptional children versus those of normal children. Journal of Research in Behavioural Sciences2009; 7(2): 175-81.

15- Gupta VB. Comparison of parenting stress in different developmental disabilities. J Dev Phys Disabil2007; 19(4): 417-25.

16- Zamani N, Zamani S, Habibi M. Comparison effectiveness of dialectic behavioral therapy and cognitive behavior therapy on emotion regulation in the mother of children with neurodevelopmental disorder. Journal of Research \& Health. In Press.

17- Jamilian HR, Malekirad AA, Farhadi M, Habibi M, Zamani N. Effectiveness of group dialectical behavior therapy (based on core distress tolerance and emotion regulation components) on Expulsive Anger and Impulsive Behaviors. Glob J Health Sci2014; 6(7): 116-23.

18- Halgin RP, Whitborne SK. Abnormal psychology, clinical perspectives on psychological disorder. New York: McGraw-Hill; 2005.

19- Zamani N, Ahmadi V, Ataie Moghanloo V, Mirshekar S. Comparing the effectiveness of two therapeutic methods of dialectical behavior therapy and cognitive behavior therapy on the improvement of impulsive behavior in the patients suffering from major depressive disorder (MDD) showing a tendency to suicide. Scientific Journal of Ilam University of Medical Sciences2014; 22(5): 45-54.

20- Sadeghi Kh, Saberi SM, Assareh M. Epidemiological study of psychiatric disorder in Kermanshah Urban residents. Andisheh va Raftar2000; $6: 22-3$ 
21- Gross JJ, John OP. Individual differences in two emotion regulation processes: Implications for affect, relationships, and well-being. J Pers Soc Psychlol2003; 85(2): 348-62.

22- Schutte NS, Malouff JM, Thorsteinsson EB, Bhullar $\mathrm{N}$, Rooke SE. A meta-anaclitic investigation of the relationship between emotional intelligence and health. Pers Individ Dif2007; 42(6): 921-33.

23- Fox HC, Hong KA, Sinha, R. Difficulties in emotion regulation and impulse control in recently abstinent alcoholics compared with social drinks. Addict Behav2008; 33(2): 388-94.

24- Gross JJ. Emotion and emotion regulation. In: Pervin LA, John OP, eds. Handbook of personality: Theory and Research (2nd. Ed). New York: Gilford press; 1999.

25- Gross JJ, Muñoz RF. Emotion regulation and mental health. Clin Psychol Rev1995; 2(2): 151-64.

26- Clarkin JF, Leavy KN, Lenzenweger MF, Kernberg OF. Evaluating three treatments for borderline personality disorder: a multiwave study. Am J Psychiatry2007; 164(6): 922-8.

27- Soler J, Pascual JC, Tiana T. Dialectical behaviour therapy skills training compared to standard group therapy in borderline personality disorder: a 3-month randomised controlled clinical trial. Behav Res Ther2009; 47(5): 353-8.

28- McQuillan A, Nicastro R, Guenot F, Girard M, Lissner C, Ferrero F. Intensive dialectical behavior therapy for outpatients with borderline personality disorder who are in crisis. Psychiatr Serv2005; 56(2): 193-7.

29- Miller AL, Rathus JH, Linehan MM, Watzler S, Leigh E. Dialectical behavior therapy adapted for suicidal adolescents. J Psychiatr Pract 1997; 3(2): 78-86. 30- van den Bosch LM, Verheul R, Schippers GM, van den Brink W. Dialectical behavior therapy of borderline patients with and without substance use problems. Implementation and long-term effects. Addict Behav2002; 27(6): 911-23.

31- Karbalaei Mohammad Meigouni A, Ahadi H, Sharifi HP, Jazayeri SM. Effects of dialectical behavior therapy with medical therapy vs. medical therapy alone in reducing suicidal thoughts in patients with major depressive disorder and recent history of attempted suicide. Journal of Applied Psychology2001; 4(16): 25-41.

32- Gross JJ. Thompson RA. Emotion regulation: conceptual foundations. In: Gross JJ. Handbook of emotion regulation. New York: Guilford press; 2007.

33- Salehi A, Baghban I, Bahrami F, Ahmadi A. The effect of emotion regulation training based on dialectical behavior therapy and gross process model on symptoms of emotional problems. Zahedan Journal of Research in Medical Sciences 2012; 14(2): 49-55.

34- Mckay M, Wood J, Brantley J. The dialectical behavior therapy skills workbook. USA: New Harbinger publications; 2007.

35- Golman D. Emotional intelligence. New York: Bantam Books; 1995.
36- Lovibond PF, Lovibond SH. The structure of negative emotional states: Comparison of the Depression Anxiety stress Scale (DASS) with the Beck Depression and Anxiety Inventories. Behav Res Ther1995; 33(3): 335-43.

37- Antony MM, Bieling PJ, Cox BJ, Enns MW, Swinton RP. Psychometric properties of the 42-item and 21-item version of the depression anxiety stress scale in clinical group and a community sample. Psychol Assess 1998; 10(2):176-181.

38- Samani S, Jokar B. A Study on the reliability and validity of the short form of the depression anxiety stress scale (DASS-21). Journal of Social Sciences and Humanities of Shiraz University2007; 26(3):65-75.

39- Fathi Ashtiani A, Dastani M. Psychological tests: Personality and mental health. Tehran: Be'sat Publication Institute; 2009.

40- Linehan M. Dialectical behavior therapy training. 2008; Available at URL: http://www.behavioraltech. org. Accessed September 20, 2015. 